\title{
Vasohibin-1 is identified as a master-regulator of endothelial cell apoptosis using gene network analysis
}

Muna Affara ${ }^{1}$, Debbie Sanders ${ }^{1}$, Hiromitsu Araki ${ }^{2}$, Yoshinori Tamada ${ }^{3}$, Benjamin J Dunmore ${ }^{1}$, Sally Humphreys ${ }^{1}$, Seiya Imoto ${ }^{3}$, Christopher Savoie ${ }^{2}$, Satoru Miyano ${ }^{3}$, Satoru Kuhara ${ }^{4}$, David Jeffries ${ }^{5}$, Cristin Print ${ }^{6,7^{*}}$ and D Stephen Charnock-Jones s $^{1,8^{*}}$

\begin{abstract}
Background: Apoptosis is a critical process in endothelial cell (EC) biology and pathology, which has been extensively studied at protein level. Numerous gene expression studies of EC apoptosis have also been performed, however few attempts have been made to use gene expression data to identify the molecular relationships and master regulators that underlie EC apoptosis. Therefore, we sought to understand these relationships by generating a Bayesian gene regulatory network (GRN) model.

Results: ECs were induced to undergo apoptosis using serum withdrawal and followed over a time course in triplicate, using microarrays. When generating the GRN, this EC time course data was supplemented by a library of microarray data from EC treated with siRNAs targeting over 350 signalling molecules.

The GRN model proposed Vasohibin-1 (VASH1) as one of the candidate master-regulators of EC apoptosis with numerous downstream mRNAs. To evaluate the role played by VASH1 in EC, we used siRNA to reduce the expression of VASH1. Of 10 mRNAs downstream of VASH1 in the GRN that were examined, 7 were significantly upor down-regulated in the direction predicted by the GRN.Further supporting an important biological role of VASH1 in EC, targeted reduction of VASH1 mRNA abundance conferred resistance to serum withdrawal-induced EC death.

Conclusion: We have utilised Bayesian GRN modelling to identify a novel candidate master regulator of EC apoptosis. This study demonstrates how GRN technology can complement traditional methods to hypothesise the regulatory relationships that underlie important biological processes.
\end{abstract}

Keywords: Vasohibin, HUVEC, Bayesian, Gene regulatory network

\section{Background}

The explosion of systems biology in recent years, facilitated by sequencing of the human genome [1,2] and the development of high throughput methods to rapidly characterise and quantify biological systems [3-6], has promoted understanding of complex biological and pathological processes. Gene regulatory networks (GRN) represent a systems biology approach, taking advantage of the growing number of RNA abundance data sets

\footnotetext{
*Correspondence: dscj1@cam.ac.uk; c.print@auckland.ac.nz

${ }^{6}$ Department of Molecular Medicine and Pathology, School of Medical

Sciences, University of Auckland, Auckland, New Zealand

'Department of Obstetrics and Gynaecology, University of Cambridge, The

Rosie Hospital, Robinson Way, Cambridge CB2 OSW, U.K

Full list of author information is available at the end of the article
}

generated by modern high throughput methods such as microarrays or RNAseq, to holistically model interactions between molecules in cells and tissues. GRN are usually displayed as directed graphs - nodes represent mRNA abundance and edges represent some form of regulatory relationship between the nodes. The reverse engineering of GRN from gene expression data has been used to understand molecular interactions in both bacterial and lower eukaryotic organisms, as well as in more complex mammalian systems. GRN employ simple correlation [7] or Boolean [8] methods, algorithms based on mutual information $[9,10]$ as well as Bayesian methods. Computational frameworks have been generated to simultaneously perform several types of GRN analysis [11]. 
Bayesian GRN are in theory especially powerful for inference of causal relationships between mRNAs in noisy microarray data [12,13]. In Bayesian GRN, the probability of the abundance of each mRNA node is modelled using a function that takes as its inputs the abundance of the node's parent mRNAs. The edges in a Bayesian GRN can represent hidden protein, non-coding RNA or metabolite-based regulatory relationships [14]. Therefore, Bayesian GRN can in theory capture information about a subset of the complex cellular regulatory circuitry [15]. Many GRN developed to date have had a 'scale free' structure [10,16], in which a small number of "hub" RNAs can be identified that are connected to large numbers of downstream RNAs in the networks. These hub RNAs are candidate master-regulators of transcription and other cellular processes. Their identification is based on relationships in the data between the hub RNA and their downstream RNAs in the GRN structure, which are usually referred to as "children". Therefore, the amount of data behind the identification of hub RNAs is much greater than the amount of data behind the identification of individual edges, and correct identification of hubs may be easier in theory than the correct identification of individual edges.

Apoptosis is pivotal for normal EC function [17], and the dysregulation of endothelial apoptosis is a key step in the development of numerous pathologies [18], including cardiovascular disease [19-21] and tumourogenesis [22-25]. Understanding the regulatory events occurring during this process in a holistic manner may provide insight into normal vascular development and maintenance, as well as vascular pathologies. Although there has been extensive characterisation of the EC proteins involved in apoptotic pathways [26-28], there have been fewer investigations into regulation of the transcriptome in ECs undergoing apoptosis [17,29].

To begin to address this issue, our group have previously used Bayesian GRN to identify molecular interactions involved in survival factor deprivation (SFD)induced EC apoptosis and cell cycle arrest [18]. This previous study used micorarray data over a triplicated eight time point SFD time course. Previous studies have illustrated the value of supplementing time series data with gene disruption data (e.g. [30]). Since at the time we were especially interested in regulation of the cell cycle, in this previous work the time series data was supplemented by eight microarrays from EC cultures treated with siRNAs targeting molecules associated with the cell cycle. This analysis identified several GRN master regulator RNAs including the $\gamma$-amino butyric acid (GABA)Receptor Associated Protein (GABARAP) [18].

In theory, the greater the volume of high-quality siRNA data used to supplement time course data, and the broader the range of RNAs targeted by the siRNAs, the more likely it is that accurate predictions can be made by GRN. Therefore, in this current study we have expanded our previous analysis by combining triplicated eight time point SFD time course data with a much larger library of EC siRNA disruptant microarray data, which was generated from the knockdown of 351 different mRNA transcripts that encode proteins with a broad range of functions in EC [11]. This expanded analysis identified numerous GRN master regulators, many of which were already known to play important roles in EC biology. However, we noted one major master regulator RNA named Vasohibin-1 (VASH1) that had not at the time been extensively studied in EC apoptosis. Therefore, we investigated the function of VASH1 in regulating mRNA abundance and in the process of EC apoptosis. We targeted VASH1 using siRNA and then used quantitative polymerase chain reaction (PCR) to examine the abundance of 10 of the 31 mRNAs directly downstream of VASH1 in the GRN. 7 of these 10 mRNAs were significantly up- or down-regulated in the direction predicted by the GRN when VASH1 expression was reduced. We also show that VASH1 is required for the apoptotic response in EC treated with SFD.

\section{Methods}

\section{Cell culture and siRNA transfection}

Umbilical cords were collected with written informed maternal consent and the approval of the Cambridge (UK) Research Ethics Committee. Human Umbilical Vein ECs (HUVECs) were isolated by collagenase digestion, as previously described [31]. Cells were cultured in fully supplemented media without antibiotics (basal EBM-2 with a propriety mix of heparin, hydrocortisone, epidermal growth factor, fibroblast growth factor, vascular endothelial growth factor, $2 \%$ foetal calf serum (FCS) Lonza, Cambridge, UK), at $37^{\circ} \mathrm{C} / 5 \% \mathrm{CO}_{2}$. To carry out siRNA transfection, HUVEC pools consisting of 10 biological isolates (of equal contribution) were prepared using passage 3 cultured cells. The HUVEC pools were plated in 6-well plates at $2.5 \times 10^{5}$ per well and left for $24 \mathrm{hrs}$ until approximately $70 \%$ confluent. siRNA transfection was carried out using pools of four siRNA duplexes from Dharmacon Inc (Lafyette, Colorado, USA) and the SiFectamine transfection reagent (ICVEC, London, UK) according to the manufacturer's instructions.

\section{RNA processing and microarray preparation}

RNA was extracted using TRIzol ${ }^{\circledR}$ reagent (Invitrogen, London, UK). RNA quality was assessed using the Agilent 2100 bioanalyser. Biotin labelled cRNA was generated and hybridised on the CodeLink Human Uniset $20 \mathrm{~K}$ microarrays following the manufacturer's instructions (Applied Microarrays, Tempe, Arizona, USA, formally supplied by GE Healthcare). 


\section{Quantitative PCR}

cDNA was synthesised from $1 \mu \mathrm{g}$ of total RNA using the QuantiTect reverse transcription kit (Qiagen, Crawley, UK), following the manufacturers protocol. Quantitative PCR was carried out using an ABI 7700 sequence analyser (Applied Biosystems, Warrington, UK). Reactions were performed using the Applied Biosystems universal master mix according to the manufacturers instructions. The Taqman probe / primers used were: VASH1 (Hs00208609_m1), SOX18 (Hs00746079_s1), PTX3 (Hs00173615_m1), FAM78A (Hs00604618_m1), PPARA (Hs00231882_m1), SLC7A2 (Hs00952727_m1), BDNF (Hs00542425_s1), MTSS1 (Hs00207341_m1), BTG2 (Hs00198887_m1), TNFSF-12 (Hs00356411_m1), FLT4 (Hs01047677_m1) and NTRK2 (Hs00178811_m1), all from Applied Biosystems.

\section{Previosuly generated microarray datasets used in this study}

The siRNA targeting of 351 different mRNA transcripts, chosen for their importance in EC biology, including transcription factors, signalling molecules, receptors and ligands is described by Hurley et. al. [11]. The microarray data from these 351 siRNA experiments is available from Gene Expression Omnibus, reference GSE27869.

The generation of triplicated microarray data from an eight time point HUVEC SFD time course has been described previously [18]. Briefly, HUVEC RNA was extracted at time points $0,0.5,1.5,3,6,9,12$ and 24 hours after survival factor withdrawal (i.e. transfer from complete media to basal EBM-2 media with no supplements apart from $2 \%$ charcoal stripped serum), and hybridised onto CodeLink Human Uniset 20K microarrays. The raw and normalised triplicate time course microarray data has been deposited in Gene Expression Omnibus, accession number GSE23067.

\section{Data processing}

CodeLink microarray quality was assessed using the CodeLink Expression analysis software v4.0. The array data were filtered to remove probes that did not contain "Good" flags in $90 \%$ of the arrays, as measured by the CodeLink Expression analysis software. The log base2 $(\log 2)$-transformed apoptosis time course data and 351 siRNA disruptant data were then both normalised using the Loess method [32,33]. For the disruptant dataset $\log 2$ ratios against a virtual median array were calculated and these ratios were then $\mathrm{z}$-transformed within each microarray prior to network inference.

For the SFD time course data, we selected transcripts concordantly regulated in abundance across the timecourse to used for GRN generation as previously described [18]. Briefly, log ratios between each time point and the first time point were calculated for all transcripts. For each transcript at each time point z-scores were then calculated by subtracting the $\log 2$ ratios from the mean of $\log 2$ ratios for that time point, and dividing by the standard deviation of $\log 2$ ratios for that time point. Transcripts were then selected that had $-2 \leq \mathrm{z} \leq+2$ at $\geq t w o$ adjacent time points in the triplicate data set. This analysis was repeated using the last time point instead of the first time point, and the union of the RNA lists prodced by the analyses that used the first and last timepoints was taken as the final list of concordantly expresed RNAs. For comparison to this z-score method, ANOVA was used to identify RNAs significantly differntially expressed at two adjacent time points relative to either the first or last time point, and the empirical Bayes method of Tai and Speed [34] was also applied. In addition, a statistically more complex method was used to identify RNAs significantly differntially expressed across the timecourse; generalised estimating equations with a Markov correlation model were fitted to the timecourse data. Contrasts were used to identify linear relationships and quadratic trends within the data using Matlab's GEEQBOX toolbox (http://www. mathworks.com/products/matlab/). Thresholds for concordant regulation were set using an absolute linear coefficient of $>21$ (and linear q value $<0.01$ ) OR an absolute quadratic coefficient of $>7$ (and q value $<0.01$ ).

All other bioinformatic manipulations used the R software package, (http://www.R-project.org), and unless otherwise stated, multiple testing corrections were applied using the Benjamini and Hochberg method. Gene ontology/pathway enrichment analyses were carried out using Fatigo software [35], GeneSetDB [36], GATHER (http://gather.genome.duke.edu/) and IPA (Ingenuity systems, www.ingenuity.com).

\section{Apoptosis bayesian GRN generation and analysis}

Apoptosis Bayesian networks were generated using the methods of [37], with some modifications. Given the relative sizes of the time-course and siRNA data sets, a dynamic GRN generated from the time-course data was used as a prior for GRN generated from the siRNA data as described [37].

When estimating the time-course GRN from the apoptosis time course microaray data, a method of bootstrapping was applied to the data. With 8 time points (obtained 0, 0.5, 1.5, 3, 6, 9, 12 and 24hrs after serum withdrawal) and 3 replicate microarray time course experiments, there are $3^{8}=6561$ possible combinations to create combinatorial apoptosis time course datasets. With such a large number of combinations, it is not computationally viable to fit regression curves through all combinations. Therefore the time course data used for network estimation was generated from the random resampling of 25 of the possible 6561 combinations as 
follows: Let $D$ be the combinatorial time course data of all genes. If $D(c)$ is the 8 time points, with each time point consisting of one of 3 replicates, then $D(c)$ can be randomly resampled with replacement 25 times from the 6561 combinations so that $D(c)(1 \leq c \leq 6561)$. The bootstrap sample can therefore be defined as $D^{*}=$ $\left\{D^{*}(1), \ldots ., D^{* *}(25)\right\}$. Using this sample of $8 \times 25=200$ randomly resampled microarrays, the apoptosis GRN was estimated. This bootstrapping procedure was repeated 100 times to generate 100 different GRNs; $\hat{G}_{T}^{* \prime} 1, \ldots . \hat{G}_{T}^{* 100}$, where $\hat{G}_{T}^{*}{ }_{T}^{B}$ is the estimated graph based on the $B$-th bootstrap sample. To estimate the reliability of the edges to be used as prior information, the bootstrap probability of each edge was calculated as follows: the reliability of the edge between the $i$-th gene to the $j$-th gene (termed the bootstrap probability) is $z_{i j}^{(1)}=$ $\left|\left\{B \mid e(i, j) \in \hat{G}_{T}^{* B}, B=1, \ldots, 100\right\}\right| / 100$. A bootstrap probability threshold value was set at $\mathrm{P}=0.8$ and only those edges that passed this threshold value were included in the prior, $Z_{1}$.

As described [37], a second prior (named the "array prior", $\mathrm{Z}_{2}$ ), was also generated. This prior was based on the up- or down-regulation of the abundance of all mRNAs, represented as z-scores, analysed by the microarrays following siRNA-medaited targeting of the 351 genes.

Priors $Z_{1}$ and $Z_{2}$, were used when inferring a static Bayesian network based on the disruptant dataset [37]. Again bootstrap resampling of the microarrays (100 times with replacement) was applied to improve the reliability of edges included in the final network. The GRNs were viewed and analysed using Cell Illustrator 5.0, freely available software that can be downloaded from http://www.cellillustrator.com.

\section{Quantification of apoptosis}

Passage 3 HUVEC pools comprising equal numbers of cells from 10 independant isolates were plated at $5 \times 10^{3}$ cells per well in a 96-well plate and cultured for $24 \mathrm{hrs}$ before siRNA transfection. Three different pools of 10 isolates were used for each assay. Cells were then left for a further $24 \mathrm{hrs}$ before treatment with either survival factor deprived conditions of basal media without supplements (EBM-2) or fully supplemented media without antibiotics (EGM-2) for 24 hrs. Active caspase-3 and -7 were quantified using the Caspase-Glo 3/7 assay system, following the manufacturer's instructions (Promega, Southampton, UK). The ADP:ATP ratio was calculated using the Apo Glow assay (Lonza, Cambridge, UK), according to the manufacture's protocol. Assays were carried out using a Fluostar Optima luminometer (BMG Labtech, Aylesbury, UK). Statistical analysis was carried out using a paired two-tailed t-test.

\section{Results}

Gene selection methods for generating a bayesian GRN to model EC apoptosis

For GRN modelling, we first identified mRNA transcripts that were significantly regulated over the timecourse of EC apoptosis. A z-score-based method for analysis of timecourse data that we have reported previously ([18] and see Methods) identified 486 significantly regulated transcripts. We analysed these 486 RNAs using the GeneSetDB web tool [36] with the Gene Ontology (GO) and WikiPathways databases. The RNAs were significantly enriched for four main categories of annotation: (i) 'cell cycle' (GO:0007049, 87 RNAs, p<0.0001 and WikiPathways:WP179, 30 RNAs, $\mathrm{p}<0.0001$ ), (ii) 'response to stress' (GO:0006950, 39 RNAs, $\mathrm{p}<0.0001$ ), (iii) 'apoptosis' (Wikipathways:WP254, 7 RNAs, $\mathrm{p}=0.016$ ) and (iv) 'immune response' (GO:0006955, 21 RNAs, $\mathrm{p}<0.0001)$.

\section{Bayesian GRN inference}

In an attempt to better understand the relationships between the mRNAs concordantly regulated in abundance during SFD, a Bayesian GRN was inferred. In theory, the more data that GRN are based on, the the more accurate their predictions can be. Therefore, for GRN generation we used a combination of the SFD timecourse data and a library of 351 siRNA disruptant microarrays. A total of 694 RNAs were used for GRN generation; the union of the 486 RNAs concordantly regulated in abundance during SFD and the 351 RNAs that were targeted by siRNA. The methodology used to generate this Bayesian network has been previously described [37] and is illustrated in Figure 1. An xml file describing this GRN can be found in Additional file 1, which can be viewed using the freely available software Cell Illustrator, and a text file listing parent and child genes for the network edges is given in Additional file 2.

\section{Identification of GRN hubs}

Hubs are highly connected nodes in GRNs and are candidate master regulators within the network structure. In a directional GRN such as the Bayesian networks generated here, they can be identified on the basis of having large numbers of downstream children. The distribution of the number of children of all nodes in the GRN is shown in the histogram in Additional file 3: Figure S1. A list of the 50 hub genes with the largest number of children (all with $\geq 20$ children) in the network is shown in Additional file 4. Given that the set of RNAs used for GRN inference was already enriched for cell cycle and stress response functions as described above, it is not surprising that many of the GRN hubs appeared to be involved in these processes. Only 53 of the 351 genes that were targetted by siRNA knock-down to generate the prior microarray data were significantly concordantly 


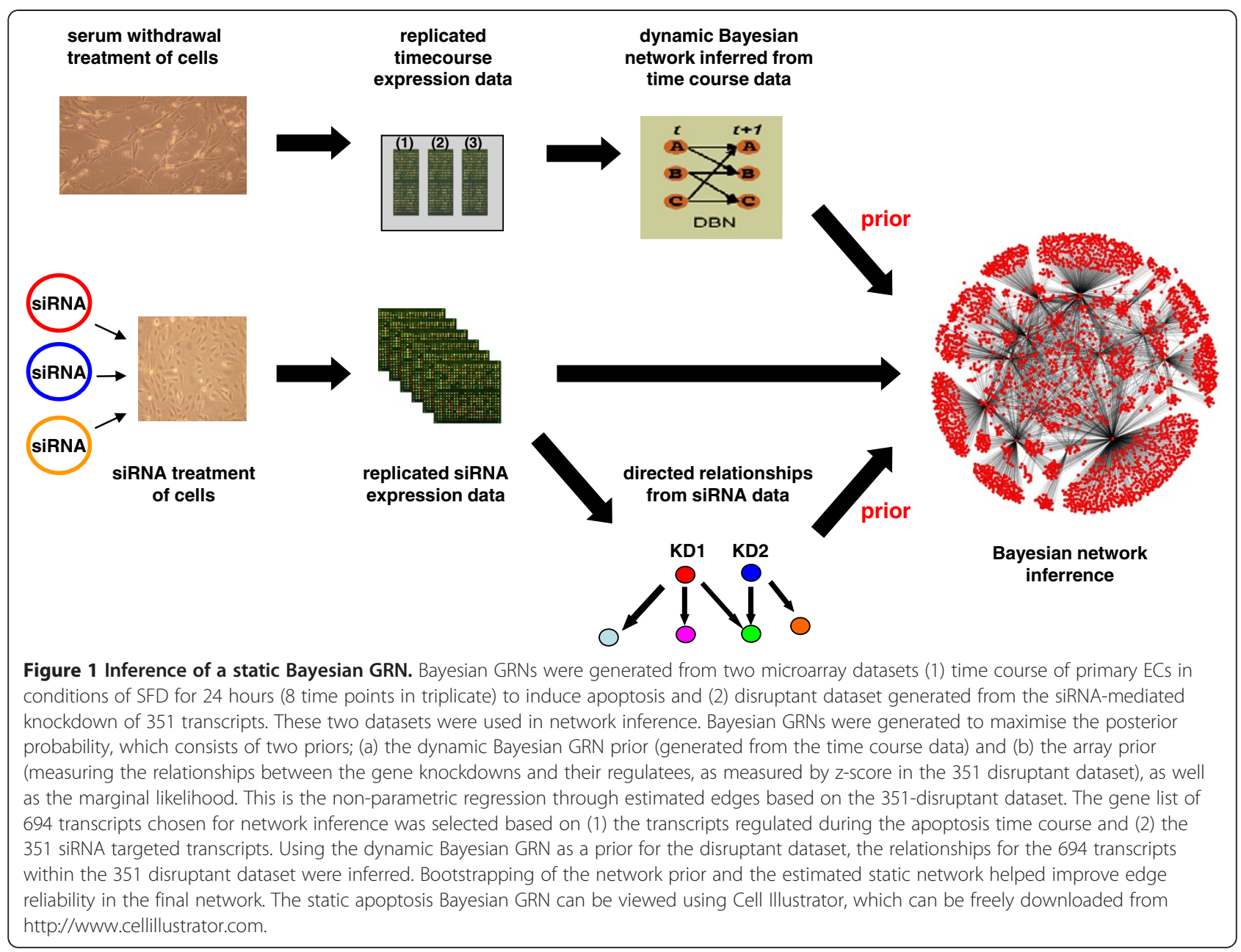

regulated in abundance over the survival factor deprivation timecourse (according to the same z-score criteria that were used to select the 486 RNAs). Of these 53 RNAs, 10 are in the list of the to 50 hubs (ranked by the number of downstream children).

We looked specifically at apoptosis-associated RNAs in the GRN. Analaysis using the Fatigo software (http:// www.fatigo.org/) identified 505 probes in the Codelink array data that encoded proteins involved in apoptosis, 58 of these probes were included as nodes in the apoptosis gene network (highlighted in Additional file 5); two of these (HSPE1 and BUB1B, with 28 and 23 children respectively) were found in the top 50 hubs ranked by number of downstream network children.

We also looked specifically at cell cycle-associated RNAs in the GRN, since SFD induces cell cycle arrest in addition to programmed cell death [29]. Of the 596 transcripts on the Codelink array associated with cell cycle regulation, 109 of these were included as nodes in the network, with 9 located within the top 50 network hubs ranked by number of downstream children.

\section{Downstream children of some GRN Hubs share common} functions

We assessed whether any of GRN hubs had downstream children significantly enriched for specific biological functions by comparing the downstream children of each hub to the datatbases GO, KEGG and Transfac using the GATHER web tool. The results of this analysis are shown in Additional file 4 and examples are given below. The hub transcript BLM ( $7^{\text {th }}$ largest hub, 33 children) encodes a DNA helicase that is important for mitotic DNA replication and DNA repair, and is mutated in a broad range of cancers [38]. 16 of the 31 children of $B L M$ encoded proteins involved in aspects of cell cycle regulation (GO:0008283, GATHER Bayes Factor $=22.3$, $\left.\mathrm{p}=3 \times 10^{-9}\right)$. Another hub gene encodes the cyclin dependent kinase-binding protein $C K S 1 B$, which has 16 of its 49 GRN children associated with cell cycle control (GO:0007049, GATHER Bayes Factor $=7.5 \mathrm{p}=0.007$ ). The hub KNTC2 encodes a kinetochore complex component that functions as a spindle checkpoint signalling molecule, and has 10 of its 18 children associated with 
the cell cycle (GO:0007049, GATHER Bayes Factor $=16$ $\left.\mathrm{p}=7.5 \times 10^{-7}\right)$. Another hub is GRN, encoding the granulin glycoprotein, a secreted regulator of cell growth and survival. 10 of GRN's 28 children are associated with the response to cell stress (GO:0006950, GATHER Bayes Factor $=8.6 \mathrm{p}=0.0005)$, and 5 with the regulation of apoptosis (GO:0042981, GATHER Bayes Factor $=5.3$ $\mathrm{p}=0.004)$. The children emenating from the apoptosisassociated hubs HSPE1 and BUB1B mentioned above also shared comon functions, however the degree of enrichment for these functions was not statistically significant. Four of the 28 children of $B U B 1 B$ were involved in cell cycle regulation (CDC16, TPX2, NUSAP and BUB1) and two in the regualtion of apoptosis (PIK3R1 and CTNAL1). Similarly, three of the 23 children of HSPE1 were involved in cell cycle processes (RAD1, E2F4 and $M C M 2)$ and two with cell death (TBP and FOSL2).

\section{Identification of a novel GRN hub gene for further study}

Evaluation of the literature revealed that several of the most highly connected hubs (such as CKS1, 49 children and $M D K, 36$ children), already had well-characterised roles in cellular proliferation and apoptosis [39-41]. In contrast, for VASH1 ( $9^{\text {th }}$ ranked hub with 31 children) there was no literature characterising its involvement in EC apoptosis or cellular proliferation at the time of this study. Several of the predicted GRN children of VASH1 are involved in the regulation of angiogenesis (e.g. FLT4, BDNF, TIE1), apoptosis (e.g. TNFSF12, PPAR- $\alpha, C D C 2 L 6$ ) and cell division (e.g. BTG2, CDKN1C). Therefore, given that the purpose of this current study was to identifiy novel regulators of EC apoptosis and since VASH1 had previously been identified as a negative regulator of angiogenesis $[42,43]$ (suggesting a possible role in EC apoptosis), VASH1 was selected as a candidate for further investigation. Due to resource limitations no other uncharacterised hubs were investigated in this study. Figure 2 illustrates the positioning of the VASH1 hub in the GRN, the 31 children eminating from this hub, and its expression profile over the SFD time course.

VASH1 would not have been prioritised as a candidate gene for further study by using traditional statistical methods that quantified the degree and variance of concordant regulation of abundance over the SFD time course. For example, when the concordant regulation of RNAs were ranked using the $\mathrm{z}$-score method or using traditional ANOVA analysis to compare expression in the first and last timepoints to all other timepoints, VASH1 was ranked 71st and 63rd, respectively. The empirical Bayes method of Tai and Speed using the Hotelling $T^{2}$-statistic [34] ranked VASH1 as 286th in terms of evidence of non-constant temporal expression. A more sophisticated method was also used, in which generalised estimating equations (with Markov correlation models) were fitted to the SFD time course data. From this regression model contrasts were used to identify linear relationships and quadratic trends within the data, and VASH1 ranked 175th.

\section{Independent validation of directed edges emanating from the VASH1 hub}

To evaluate the RNAs hypothesised by the GRN to be downstream of the VASH1 hub, 10 of the 31 children were selected for independent validation using siRNA knock-down and quantitative PCR. The selection was based on known biological importance and reagent availability. The left column of panels in Figure 3 illustrates the transcript profiles of VASH1 and selected children over the SFD time course. In the case of MTSS1 (3a) and SOX18 (3d), the children are positively co-regulated with VASH1 over the apoptosis time course. In contrast, $B D N F(3 \mathrm{~g})$ and SLC7A2 (3j) are negatively co-regulated with VASH1 over the time course. Correlation analysis across the 351 siRNA disruptant dataset revealed that all 10 children correlated with VASH1 (correlation coefficients range from $0.5-0.8$ ); the relationships between

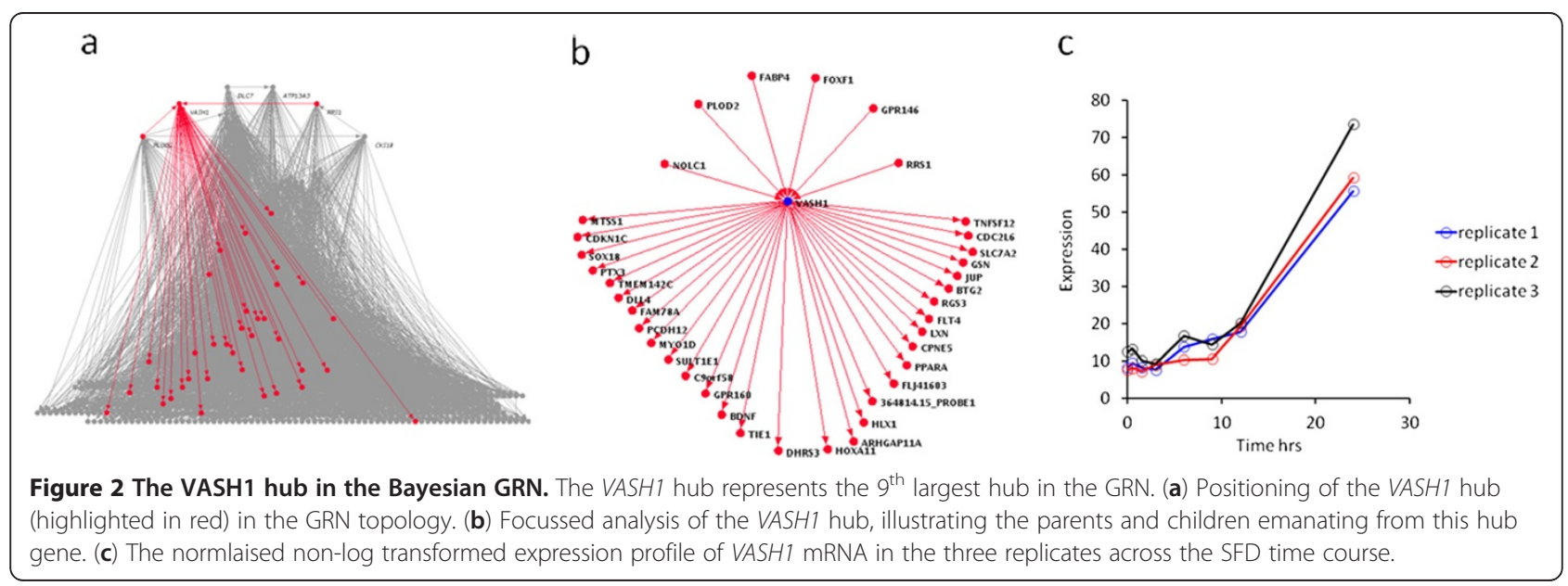




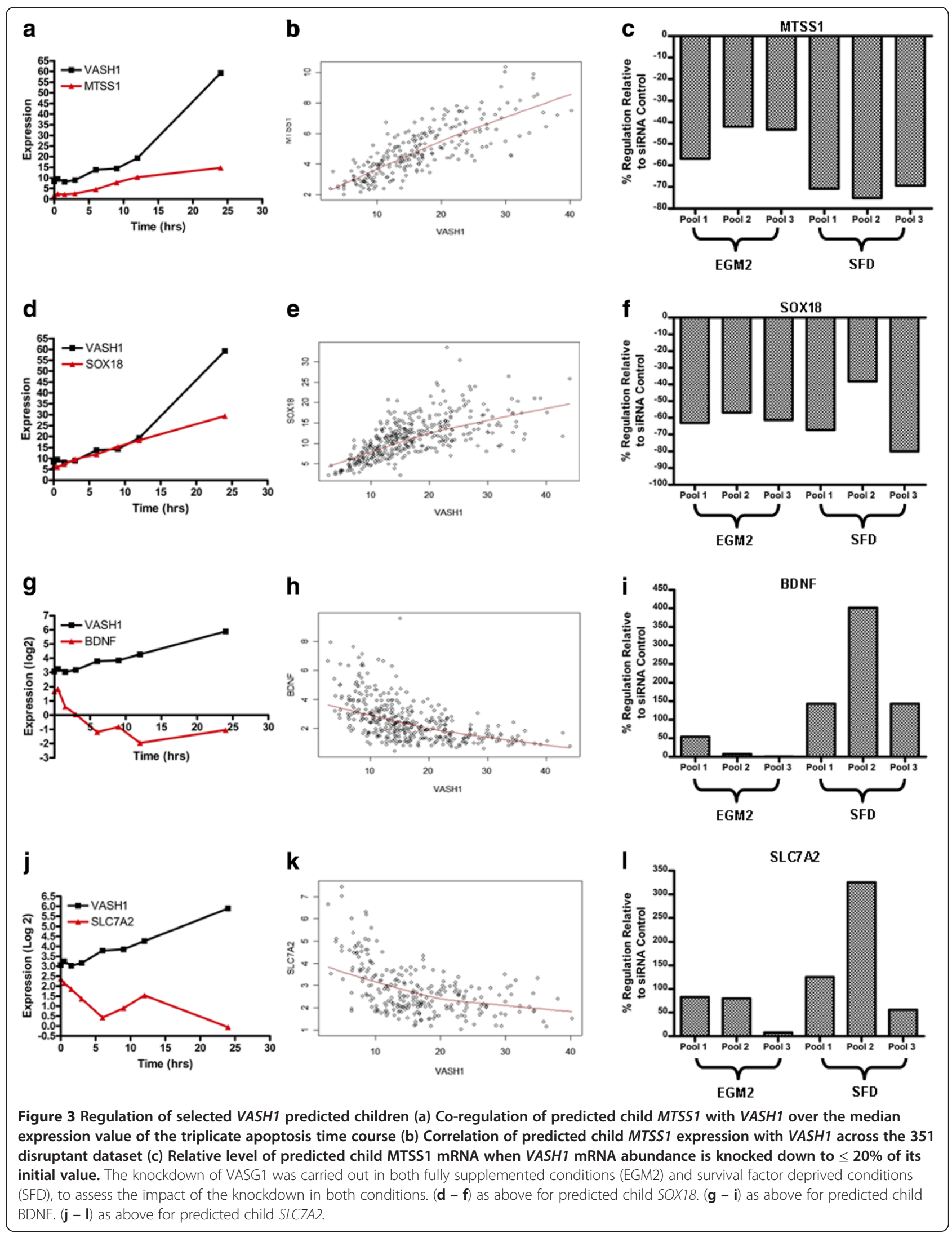


VASH1 and these downstream children across the 351 siRNA disruptant microarrays are illustrated in scatter plots in the middle panels of Figure 3. VASH1-SLC7A2 and $V A S H 1-B D N F$ associated with negative correlation, while the remaining children correlate positively with VASH1 (Figure 3b, e, h and $\mathrm{k}$ ). This correlation across the 351 siRNA disruptant dataset concurred with the co-regulation observed over the apoptosis time course in Figure $3 a, d, g$ and $j$.

Using siRNA to knockdown VASH1 mRNA to $\leq 20 \%$ of its initial level appeared to significantly regulate 7 of the 10 transcripts tested, in the direction predicted by the GRN (Table 1). For example, MTSS1 and SOX18, which were positively correlated with $V A S H 1$, were downregulated after knockdown of VASH1 (Figure 3c and 3f respectively). In contrast, but as predicted by the GRN, knockdown of $V A S H 1$ resulted in the up-regulation of $B D N F$ and SLC7A2, which were negatively correlated with VASH1 (Figure 3i and Figure 3l). TNFSF12, PTX3 and $F A M 78 A$ did not show a clear result due to variable regulation between EC replicate pools.

\section{Regulation of apoptosis by $\mathrm{VASH} 1$}

To evaluate whether the VASH1 hub is involved in the process or regulation of EC apoptosis, siRNA was used to knockdown $V A S H 1$ in three different pools of 10 HUVEC isolates for 24 hours before treatment with SFD to induce apoptosis. After the 24 hour anti-VASH1 siRNA incubation, VASH1 mRNA abundance was reduced to $\leq 20 \%$ of its initial level (Figure 4 ). Following SFD there was a mean of 2.2 fold ( $\mathrm{t}$-test, $\mathrm{p}=0.0009$ ) less active caspase- 3 and -7 in the VASH1 knockdown EC compared to the EC teated with non-targeting siRNA

Table 1 Fold change and P values of VASH1 and its predicted children in fully supplemented conditions and SFD conditions, after knockdown of VASH1

\begin{tabular}{|c|c|c|c|c|}
\hline & \multicolumn{2}{|c|}{$\begin{array}{l}\text { Fully Supplemented Media } \\
\text { (EGM2) }\end{array}$} & \multicolumn{2}{|c|}{$\begin{array}{c}\text { Survival Factor deprived } \\
\text { Media (SFD) }\end{array}$} \\
\hline & Fold Change & P Value & Fold Change & P Value \\
\hline VASH1 & -7.59 & 0.0022 & -17.50 & 0.0025 \\
\hline BDNF & 3.90 & 0.4138 & 12.52 & 0.0339 \\
\hline BTG2 & -1.65 & 0.1592 & -3.10 & 0.0005 \\
\hline FAM78A & -1.97 & 0.0577 & -0.69 & 0.5752 \\
\hline FLT4 & -1.99 & 0.0404 & -3.67 & 0.0364 \\
\hline MTSS1 & -1.82 & 0.0128 & -3.97 & 0.0708 \\
\hline PPARA & -1.92 & 0.0962 & -2.79 & 0.0034 \\
\hline PTX3 & -1.04 & 0.4932 & 0.54 & 0.6942 \\
\hline SLC7A2 & 1.96 & 0.1087 & 2.30 & 0.0237 \\
\hline SoX18 & -1.41 & 0.5587 & -3.81 & 0.0002 \\
\hline TNFSF12 & 0.46 & 0.5499 & -1.82 & 0.0223 \\
\hline
\end{tabular}

$P$ values are from a paired $t$ test.

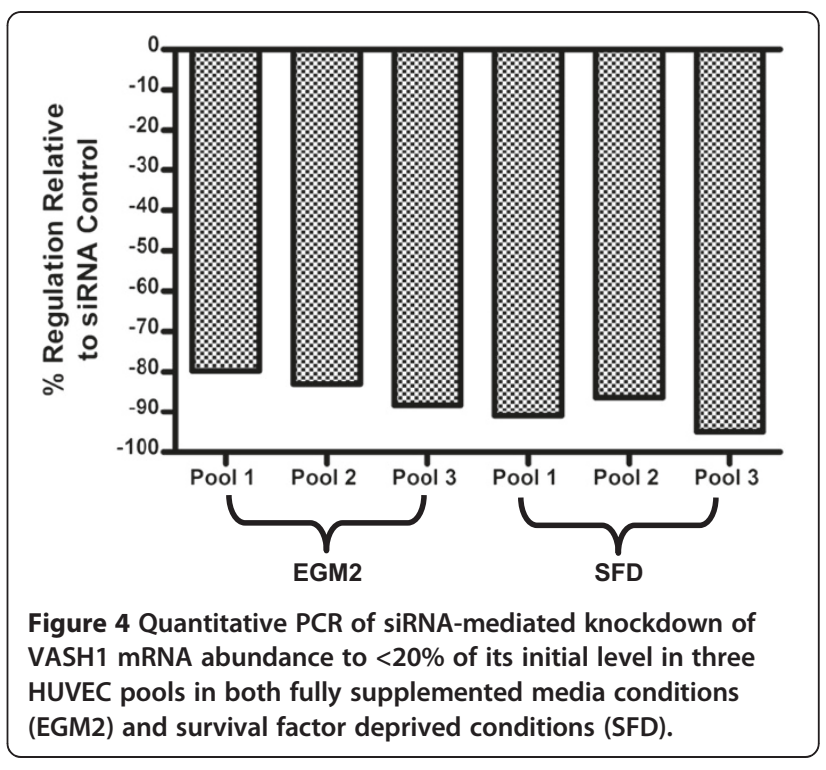

controls (Figure 5a). Repetition of this assay in two additional pools of HUVEC isolates in which VASH1 was once again knocked down to $\leq 20 \%$ of its initial level produced a similar result - following SFD there was on average 1.8 fold $(\mathrm{p}=0.03$ ) less active caspase-3 and -7 following VASH1 knockdown than in control cells (data not shown). The observed level of active caspase- 3 and -7 in HUVEC in fully supplemented conditions was similar in VASH1 knockdown and control cells (data not shown).

The activation of caspase- 3 and -7 only represents one part the complex process of apoptosis. Since apoptosis is an energy driven process, the ADT:ATP ratio was also calculated in the same HUVEC pools. A marked reduction in the mean ADP:ATP ratio was observed in the VASH1 knockdown EC relative to the siRNA control EC following SFD in two independent experiments (12.4 fold, $\mathrm{P}=0.004$ and 3.4 fold, $\mathrm{P}=0.005$, respectively, Figure 5b). Again, no significant difference was observed between the VASH1 knock down EC and controls in fully supplemented conditions (data not shown). Taken together, these results suggest that VASH1 may play a significant role in SFD-induced apoptosis of HUVECs.

The inverse expression relationship between VASH1 and its validated child $B D N F$, and the known role of BDNF as a survival factor, suggests the hypothesis that up-regulation of $B D N F$ when VASH1 is knocked down may promote survival in these cells. However, the treatment of the HUVEC pools with $100 \mathrm{nM}$ recombinant $B D N F$ at 24 hours post-transfection (at the same point as treatment with either fully supplemented media or SFD conditions), did not induce significant rescue from SFD-induced cell death, as measured by both the quantification of active caspase- 3 and 7 and the ADP:ATP ratio (data not shown). 

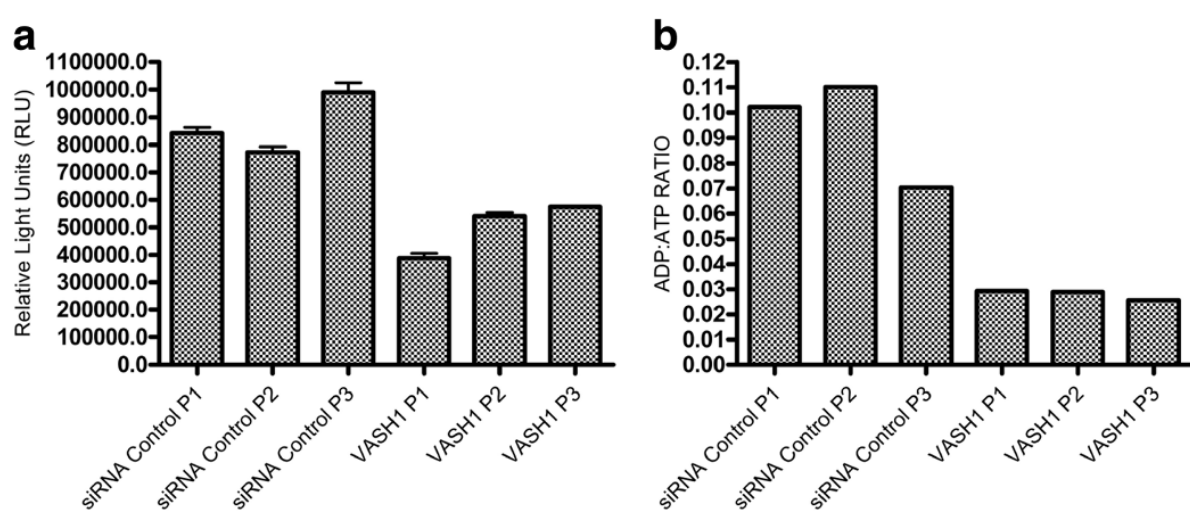

Figure 5 (a) Quantification of active caspase-3 and -7 in three independent pools of 10 HUVEC isolates treated with either a nontargeting siRNA for $\mathbf{4 8 h r s ~ o r ~ s i R N A ~ a g a i n s t ~ V A S H 1 ~ f o r ~} \mathbf{4 8} \mathbf{~ h r s . ~ H U V E C s ~ w e r e ~ t r e a t e d ~ 2 4 h r s ~ p o s t ~ t r a n s f e c t i o n ~ w i t h ~ s u r v i v a l ~ f a c t o r ~ d e p r i v e d ~ ( S F D ) ~}$ conditions for $24 \mathrm{hrs}$ before measurement. A significant difference was observed between the VASH1 knockdown and the siRNA control in the SFD condition ( $P=0.0009$, paired two tailed t-test). (b) Quantification of the ADP:ATP ratio in three pools of 10 HUVEC isolates treated with either a non-targeting siRNA for 48hrs or siRNA against VASH1 for 48hrs. HUVECs were treated 24hrs post transfection with SFD conditions for 24hrs before measurement $(P=0.02)$. $P=$ Pool.

\section{Discussion}

This study used Bayesian GRN technology and microarray data to model the regulatory interactions after serum factor deprivation of EC, which induces cell cycle arrest and apoptosis. We applied GRN analysis to combined time course microarray data following serum deprivation-induced apoptosis of EC, and a large microarray data set generated from 351 targeted EC siRNA disruptions, in order to identifiy new information about gene regulatory relationships during EC apoptosis.

\section{GRN topology and limitations}

We have used GRN analysis to identify hubs, which potentially act as master regulators of the expression of downstream RNAs in EC. For one hub, VASH1, we then confirmed the concordant regulation of a subset of downstream children as hypothesised by the GRN, and an impact on apoptosis. VASH1 protein has previously been identified as a negative feedback regulator of angiogenesis, and is induced through signalling via VEGFR2 and protein kinase $C[43,44]$. These findings are consistent with a role for VASH1 as a key regulator of EC biology, and with the position of VASH1 as a hub in the GRN with an effect on EC apoptosis.

We suggest that GRN analysis may provide a useful compliment to traditional analysis of microarray or RNAseq data, especially for identifying putative masterregulators for further study. We showed that alternative methods to identify apoptosis-associated RNAs from time course expression data were unlikely to have selected $V A S H 1$ as a candidate gene for subsequent functual analaysis. Methods based on: z-scores, ANOVA and empirical Bayes all failed to prioritise VASH1.
However, we also recognise the potential limitations of gene network analysis. Firstly, only a few of the transcripts that encode protein mediators of apoptosis are expected to be up- or down-regulated during relatively short time points of SFD-induced apoptosis [29] to a sufficint degree to be included in this analysis, and SFD is only one of many inducers of apoptosis. Therefore, only a subset of known apoptosis-associated genes are accessible to this type of GRN study. Secondly, many genes known to be important in the apoptotic process may not be "master regulators" detectable as hubs in gene networks, which requires that they rapidly regulate the abundance of large numbers of downstream RNAs. Since we used timecourse microarray data from EC cells treated with survival factor deprivation, we expected to identify only master regulators that were specifically related to the molecular processes that occur during this timecourse. To be identified as a "hub" in the gene network, both an RNA, and its downstream "child" RNAs, must be significantly concordantly regulated over the timecourse. Therefore, not all RNAs important for EC biology will be concordantly regulated during this specialised type of apoptosis, and even if they are, they will not be identified as hubs unless they rapidly regulate the abundance of large numbers downstream RNA transcripts. Thirdly, like all in silico modelling based on microarray or RNAseq data, results of special interest from GRN analysis need to be confimred using laboratory experiments as we have done here.

\section{Inference of local relationships within the network}

Several GRN methods have proven informative for identifying regulatory hubs or cohorts of co-expressed genes 
in complex eukaryotic cells, which are involved in important disease processes [10,45-47]. However, most of these methods fall short of inferring directional relationships at a local level. Therefore, having identified the VASH1 hub based on network topology, we examined the GRN predictions surrounding this hub in more detail. Using siRNA we knocked down VASH1 mRNA and determined the effect on expression levels of downstream mRNAs for ten out of VASH1's 31 GRN children. Seven out of the ten children tested were significantly up- or down-regulated in the direction predicted by the GRN (see Table 1). The lack of clear influence of VASH1 knock-down on three child-transcripts may be due to several factors: (i) Reducing VASH1 RNA may have little effect on the abundance of those gene network children of VASH1 that are strongly influenced by other parents in addition to VASH1 - the undiminished effects of these other parents would be expected to hide the effect of reducing VASH1 expression. (ii) Regulatory relationships that are not represented in the GRN may influence the expression of some of VASH1's gene network children, (iii) Despite best efforts, the effects of experimental noise and unintended model over-fitting are likely to have introduced error in the inference process. These issues are further described in our recent publications $[48,49]$. It is possible that additional siRNA data may improve the accuracy of GRNs around VASH1, which is a subject for future research.

Whether the observed level of concordance between the network predictions and the results of experimental $V A S H 1$ knockdown only surrounds the major hubs within the Bayesian network structure, or is randomly distributed throughout the network, requires further investigation. Due to resource constraints we have only evaluated a minority of edges downstream of a single hub. This is clearly not enough to draw any general conclusions about GRNs and their reliability. Given further resources, we would like to evaluate the relationships between VASH1 and the remaining 21 children that we have not yet tested, as well as the relationships between several other nodes and their children. To more completely test local network relationships we would ultimately need to simultaneously perform siRNA-mediated "knockdown" of all the gene network parents of each VASH1 child then measure the effect on $V A S H 1$ child abundance. In addition, as computational capabilities improve, it would be interesting to re-engineer GRNs with the inclusion of more of the replicate arrays and compare the reliability with that of the current network model. Nevertheless, given the early stage of this technology, the fact that only one (VASH1) of several parents of the evaluated VASH1 children was knocked down, and the fact that there are data missing from the network (due to computational constraints it contains only 694 transcripts), these current findings appear promising.

\section{Functional significance of the VASH1 gene network hub}

To investigate whether the VASH1 hub was biologically relevant during endothelial apoptosis, we used siRNAs targeted against $V A S H 1$ to reduce mRNA abundance to $<20 \%$ of its initial level and quantified the level of apoptosis in these cells under conditions of SFD relative to control cells transfected with an irrelevant siRNA. The measurement of active caspase- 3 and -7 and the ADP: ATP ratio were used as end-points and the knock-down of VASH1 conferred resistance to the pro-apoptotic stimulus of serum deprivation. This confirms a role for $V A S H 1$ in the process of EC apoptosis, and is consistent with a study published since this research was conducted that shows over expression of VASH1 induced apoptosis in proliferating human fibroblasts [50].

Although the mechanism by which VASH1 regulates EC survival is beyond the scope of this study, it is intriguing to examine the function of VASH1's GRN children in the anticipation that this may suggest how VASH1 acts. Ingenuity Pathways Analysis (IPA) suggested that the VASH1 GRN children are significantly enriched for genes associated with angiogenesis $(F D R=0.025)$, including several well-known angiogenic regulators; $B D N F$, DLL4 (delta-like 4), FLT4 (vegfr-3), PPARA, PTX3, SOX18, TIE1, and TNFSF12 (tweak). Several of the VASH1 children have previously been associated with the linked control of proliferation and apoptosis e.g. CDKN1C (p57/kip2), CDC2L6, GSN (gelsolin) and DLL4. It is interesting that 20 of the 31 VASH1 children have binding sites for members of the E2F transcription factor family in their promoters $\left(\mathrm{V} \$ \mathrm{E} 2 \mathrm{~F} 1 \_\mathrm{Q} 3, \mathrm{FDR}=0.002\right)$; experiments to assess whether E2F transactivation is regulated by VASH1 may be worthwhile.

One mechanistic hypothesis was investigated in this study. The autocrine action of BDNF in cellular processes including angiogenesis, proliferation, differentiation and survival is well documented [51-53]. Therefore, the inverse relationship between $V A S H 1$ and its validated child $B D N F$, suggests a hypothesis that upregulation of $B D N F$ when $V A S H 1$ is knocked down may promote survival. However, recombinant $B D N F$ was unable to rescue the cells from SFD induced death. It is possible that the chosen concentration of recombinant BDNF (100nM) and time point of administration were not optimal. However, the results suggest that it is unlikely that the mechanism of VASH1 action in EC apoptosis is as simple as upregulation of $B D N F$. The relatively low expression of the BDNF high affinity (TrkB) and the low affinity $\left(\mathrm{p} 75^{\mathrm{NTR}}\right)$ receptors observed in the microarray data from these cells $\left(\operatorname{TrkB}\right.$, percentile rank expression $=0.142113$ and $p 75^{N T R}$, percentile rank expression $=0.414449$ in healthy HUVEC) may also explain why exogenous BDNF was not effective.

Since this study was conducted, several publications have described the role of VASH1 in regulating EC 
proliferation, vascular tube generation and vascular maturation $[50,54][55]$, as well as a potentially wider functional role in other cell types [56]. In addition, VASH1 may interact in complex functional networks with related proteins such as VASH2 to regulate angiogensis and EC survival differently in distinct angiogenic mechanisms [57]. Whatever its mechanism of action, VASH1 appears to be associated with angiogenesis in pathology $[43,58]$, and further investigation of the molecular networks that surround VASH1 seem highly worthwhile.

\section{Conclusion}

GRN analysis is able to supplement the reductionist methods of traditional molecular biology, providing testable hypotheses about the synergistic actions and interactions of multiple molecules [59]. We therefore applied Bayesian GRN methods to further our understanding of the regulation of $\mathrm{EC}$ apoptosis and proliferation. The SFD Bayesian GRN generated in this study identified VASH1 as a candidate master regulator, which we found was functionally important during EC apoptosis. We also found that several individual directed edges emanating from VASH1 in the GRN appeared to operate in ECs. We hope that in future studies the datasets we describe here can be used by other researchers to identify additional candidate master regulators for laboratory evaluation.

\section{Additional files}

\section{Additional file 1. XML file describing the GRN.}

Additional file 2. List of the parent child relationships inferred from the GRN.

Additional file 3: Figure S1. The histiogram shows the frequency (y-axis) of number of children ( $x$-axis) in the GRN.

Additional file 4. List of the 50 hub genes with the largest number of children in the GRN.

Additional file 5. List of apoptosis associated RNA's in the Codelink microarray platform and the GRN.

\section{Competing interests}

Ther are no strong competing interests. However, for completenes we wish to disclose that: HA, YT and CS are past employees of GNI Ltd., a Japanese biotechnology company which supported the generation of microarray data used in this study. All authors have held stock or stock options in GNI Ltd. None of the authors are current employees of GNI Ltd.

\section{Authors' contributions}

All authors fullfil the criteria for authorship. MA wrote the manuscript and primarily generated the new experimental data shown here. DS, SH and BD collaborated extensively with and supervised MA in the laboratory for molecular biology and microarray studies and assisted with data analysis. HA undertook bioinformatics of siRNA array data and assisted with Bayesian network analysis. YT and SI performed Bayesian network analysis and gave advice on network inference issues. CS, SM and SK assisted with the planning and funding of the project, supervised the generation of gene network software and its use. DJ performed statistical analysis and provided statistical advise. CP and DSCJ jointly supervised MA as a postgraduate student, undertook the experimental planning of the project, oversaw and assisted with data interpretation and manuscript preparation.

\section{Authors' information}

Cristin Print and Stephen Charnock-Jones Joint senior authors.

\section{Acknowledgements}

A subset of the laboratory work described in this manuscript was funded by the company GNI Ltd. The Biotechnology and Biological Sciences Research Council (BBSRC) also supported aspects of this research. Part of CP's contribution was funded by the Health Research Council of New Zealand's International Investment Opportunities Fund. The supercomputing resource for the estimation of GRN was provided by Human Genome Center, Institute of Medical Science, University of Tokyo (http://sc.hgc.jp/shirokane.html).

\section{Author details}

'Department of Obstetrics and Gynaecology, University of Cambridge, The Rosie Hospital, Robinson Way, Cambridge CB2 OSW, U.K. ${ }^{2}$ Systems

Pharmacology Research Institute, GNI Ltd., Fukuoka, Japan. ${ }^{3}$ Human Genome Centre, Institute of Medical Science, The University of Tokyo, Tokyo, Japan. ${ }^{4}$ Graduate School of Genetic Resources Technology, Kyushu University, Fukuoka, Japan. ${ }^{5} \mathrm{MRC}$, The Gambia Unit, Fajara, The Gambia. ${ }^{6}$ Department of Molecular Medicine and Pathology, School of Medical Sciences, University of Auckland, Auckland, New Zealand. "Bioinformatics Institute, University of Auckland, Auckland, New Zealand. ${ }^{8}$ National Institute for Health Research, Cambridge Comprehensive Biomedical Centre, Cambridge, UK.

Received: 15 February 2012 Accepted: 7 December 2012

Published: 16 January 2013

\section{References}

1. Venter JC, Adams MD, Myers EW, Li PW, Mural RJ, Sutton GG, Smith HO, Yandell M, Evans CA, Holt RA, et al: The sequence of the human genome. Science 2001, 291(5507):1304-1351.

2. International Human Genome Sequencing Consortium: Finishing the euchromatic sequence of the human genome. Nature 2004 431(7011):931-945.

3. Schena M, Shalon D, Davis RW, Brown PO: Quantitative monitoring of gene expression patterns with a complementary DNA microarray. Science 1995, 270(5235):467-470.

4. Mortazavi A, Williams BA, McCue K, Schaeffer L, Wold B: Mapping and quantifying mammalian transcriptomes by RNA-Seq. Nat Methods 2008, 5(7):621-628.

5. Pezzino S, Paratore $\mathrm{S}$, Cavallaro S: Systems biology of apoptosis and survival: implications for drug development. Curr Pharm Des 2011, 17(3):190-203.

6. Cloonan N, Forrest AR, Kolle G, Gardiner BB, Faulkner GJ, Brown MK, Taylor DF, Steptoe AL, Wani S, Bethel G, et al: Stem cell transcriptome profiling via massive-scale mRNA sequencing. Nat Methods 2008, 5(7):613-619.

7. Slavov N, Dawson KA: Correlation signature of the macroscopic states of the gene regulatory network in cancer. Proc Natl Acad Sci U S A 2009, 106(11):4079-4084

8. Akutsu T, Kuhara S, Maruyama O, Miyano S: A System for Identifying Genetic Networks from Gene Expression Patterns Produced by Gene Disruptions and Overexpressions. Genome Inform Ser Workshop Genome Inform 1998, 9:151-160.

9. Butte AJ, Kohane IS: Mutual information relevance networks: functional genomic clustering using pairwise entropy measurements. Pac Symp Biocomput 2000, :418-429.

10. Basso K, Margolin AA, Stolovitzky G, Klein U, Dalla-Favera R, Califano A: Reverse engineering of regulatory networks in human B cells. Nat Genet 2005, 37(4):382-390.

11. Hurley D, Araki H, Tamada Y, Dunmore B, Sanders D, Humphreys S, Affara M, Imoto S, Yasuda K, Tomiyasu Y, et al: Gene network inference and visualization tools for biologists: application to new human transcriptome datasets. Nucleic Acids Res 2011, 40(6):2377-2398.

12. Friedman N, Linial M, Nachman I, Pe'er D: Using Bayesian networks to analyze expression data. J Comput Biol 2000, 7(3-4):601-620.

13. Hartemink AJ, Gifford DK, Jaakkola TS, Young RA: Using graphical models and genomic expression data to statistically validate models of genetic regulatory networks. Pac Symp Biocomput 2001, :422-433. 
14. Imoto S, Higuchi T, Goto T, Tashiro K, Kuhara S, Miyano S: Combining microarrays and biological knowledge for estimating gene networks via bayesian networks. J Bioinform Comput Biol 2004, 2(1):77-98.

15. Brazhnik P, de la Fuente A, Mendes P: Gene networks: how to put the function in genomics. Trends Biotechnol 2002, 20(11):467-472.

16. Babu MM, Luscombe NM, Aravind L, Gerstein M, Teichmann SA: Structure and evolution of transcriptional regulatory networks. Curr Opin Struct Biol 2004, 14(3):283-291.

17. Duval H, Harris M, Li J, Johnson N, Print C: New insights into the function and regulation of endothelial cell apoptosis. Angiogenesis 2003, 6(3):171-183.

18. Affara M, Dunmore B, Savoie C, Imoto S, Tamada Y, Araki H, Charnock-Jones DS, Miyano S, Print C: Understanding endothelial cell apoptosis: what can the transcriptome, glycome and proteome reveal? Philos Trans $R$ Soc Lond B Biol Sci 2007, 362(1484):1469-1487.

19. Zhang W, Li D, Mehta JL: Role of AIF in human coronary artery endothelial cell apoptosis. Am J Physiol Heart Circ Physiol 2004, 286(1):H354-H358.

20. Stefanec T: Endothelial apoptosis: could it have a role in the pathogenesis and treatment of disease? Chest 2000, 117(3):841-854.

21. Xia L, Ding F, Zhu JH, Fu GS: Resveratrol attenuates apoptosis of pulmonary microvascular endothelial cells induced by high shear stress and proinflammatory factors. Hum Cell 2011, 24(3):127-133.

22. Reed JC: Dysregulation of apoptosis in cancer. J Clin Oncol 1999, 17(9):2941-2953.

23. Lunt SJ: Chaudary N. Hill RP: The tumor microenvironment and metastatic disease. Clin Exp Metastasis; 2008

24. Palmero El, de Campos SG, Campos M, de Souza NC, Guerreiro ID, Carvalho $\mathrm{AL}$, Marques MM: Mechanisms and role of microRNA deregulation in cancer onset and progression. Genet Mol Biol 2011, 34(3):363-370.

25. Fabregat I: Dysregulation of apoptosis in hepatocellular carcinoma cells. World J Gastroenterol 2009, 15(5):513-520.

26. Saraste A, Pulkki K: Morphologic and biochemical hallmarks of apoptosis Cardiovasc Res 2000, 45(3):528-537.

27. Kerr JF, Wyllie AH, Currie AR: Apoptosis: a basic biological phenomenon with wide-ranging implications in tissue kinetics. Br J Cancer 1972, 26(4):239-257.

28. Hacker G: The morphology of apoptosis. Cell Tissue Res 2000, 301(1):5-17.

29. Johnson NA, Sengupta S, Saidi SA, Lessan K, Charnock-Jones SD, Scott L, Stephens R, Freeman TC, Tom BD, Harris M, et al: Endothelial cells preparing to die by apoptosis initiate a program of transcriptome and glycome regulation. FASEB J 2004, 18(1):188-190.

30. Savoie CJ, Aburatani S, Watanabe S, Equchi Y, Muta S, Imoto S, Miyano S, Kuhara S, Tashiro K: Use of gene networks from full genome microarray libraries to identify functionally relevant drug-affected genes and gene regulation cascades. DNA Res 2003, 10(1):19-25.

31. Jaffe EA, Nachman RL, Becker CG, Minick CR: Culture of human endothelial cells derived from umbilical veins. Identification by morphologic and immunologic criteria. J Clin Invest 1973, 52(11):2745-2756.

32. Schoenfeld J, Lessan K, Johnson NA, Charnock-Jones DS, Evans A, Vourvouhaki E, Scott L, Stephens R, Freeman TC, Saidi SA, et al: Bioinformatic analysis of primary endothelial cell gene array data illustrated by the analysis of transcriptome changes in endothelial cells exposed to VEGF-A and PIGF. Angiogenesis 2004, 7(2):143-156.

33. Cleveland WS: Robust Locally Weighted Regression and Smoothing Scatterplots. J Am Stat Assoc 1979, 74(368):829-836.

34. Tai YC, Speed T: A multivariate empirical Bayes statistic for replicated microarray time course data. Ann Stat 2006, 34(5):2387-2412.

35. Al-Shahrour F, Diaz-Uriarte R, Dopazo J: FatiGO: a web tool for finding significant associations of Gene Ontology terms with groups of genes. Bioinformatics 2004, 20(4):578-580.

36. Araki H, Knapp, C, Tsai P and Print C. GeneSetDB: a comprehensive metadatabase, statistical and visualisation framework for gene set analysis. FEBS Open Bio 2012, 2(2012):76-82.

37. Imoto S, Tamada Y, Araki H, Yasuda K, Print CG, Charnock-Jones SD, Sanders D, Savoie CJ, Tashiro K, Kuhara S, et al: Computational strategy for discovering druggable gene networks from genome-wide RNA expression profiles. Pac Symp Biocomput 2006, :559-571.

38. Guo RB, Rigolet $P$, Ren $H$, Zhang B, Zhang XD, Dou SX, Wang PY, Amor-Gueret M, Xi XG: Structural and functional analyses of diseasecausing missense mutations in Bloom syndrome protein. Nucleic Acids Res 2007, 35(18):6297-6310.

39. Lan Y, Zhang Y, Wang J, Lin C, Ittmann MM, Wang F: Aberrant expression of $\mathrm{Cks} 1$ and $\mathrm{Cks} 2$ contributes to prostate tumorigenesis by promoting proliferation and inhibiting programmed cell death. Int J Cancer 2008, 123(3):543-551.

40. Wang XC, Tian LL, Tian J, Wu HL, Meng AM: Overexpression of Cks1 is associated with poor survival by inhibiting apoptosis in breast cancer. J Cancer Res Clin Oncol 2009, 135(10):1393-1401.

41. Wang Y, Xing H, Tian Z, Tang K, Wang J, Xu Z, Rao Q, Wang M, Wang J: Overexpression of Midkine promotes the viability of BA/F3 cells. Biochem Biophys Res Commun 2009, 384(3):341-346.

42. Kerbel RS: Vasohibin: the feedback on a new inhibitor of angiogenesis. J Clin Invest 2004, 114(7):884-886.

43. Watanabe K, Hasegawa Y, Yamashita H, Shimizu K, Ding Y, Abe M, Ohta H, Imagawa K, Hojo K, Maki H, et al: Vasohibin as an endothelium-derived negative feedback regulator of angiogenesis. J Clin Invest 2004, 114(7):898-907.

44. Shimizu K, Watanabe K, Yamashita H, Abe M, Yoshimatsu H, Ohta H, Sonoda $H$, Sato $Y$ : Gene regulation of a novel angiogenesis inhibitor, vasohibin, in endothelial cells. Biochem Biophys Res Commun 2005, 327(3):700-706.

45. Chen Y, Zhu J, Lum PY, Yang X, Pinto S, MacNeil DJ, Zhang C, Lamb J, Edwards S, Sieberts SK, et al: Variations in DNA elucidate molecular networks that cause disease. Nature 2008, 452(7186):429-435.

46. Emilsson V, Thorleifsson G, Zhang B, Leonardson AS, Zink F, Zhu J, Carlson S, Helgason A, Walters GB, Gunnarsdottir $S$, et al: Genetics of gene expression and its effect on disease. Nature 2008, 452(7186):423-428.

47. Lamb J, Crawford ED, Peck D, Modell JW, Blat IC, Wrobel MJ, Lerner J, Brunet JP, Subramanian A, Ross KN, et al: The Connectivity Map: using gene-expression signatures to connect small molecules, genes, and disease. Science 2006, 313(5795):1929-1935.

48. Wang L, Hurley DG, Watkins W, Araki H, Tamada Y, Muthukaruppan A, Ranjard L, Derkac E, Imoto S, Miyano S, et al: Cell cycle gene networks are associated with melanoma prognosis. PLoS One 2012, 7(4):e34247.

49. Hurley D, Araki H, Tamada Y, Dunmore B, Sanders D, Humphreys S, Affara M, Imoto S, Yasuda K, Tomiyasu Y, et al: Gene network inference and visualization tools for biologists: application to new human transcriptome datasets. Nucleic Acids Res 2012, 40(6):2377-2398.

50. Kern J, Bauer M, Rychli K, Wojta J, Ritsch A, Gastl G, Gunsilius E, Untergasse G: Alternative splicing of vasohibin-1 generates an inhibitor of endothelial cell proliferation, migration, and capillary tube formation. Arterioscler Thromb Vasc Biol 2008, 28(3):478-484.

51. Pearse RN, Swendeman SL, Li Y, Rafii D, Hempstead BL: A neurotrophin axis in myeloma: TrkB and BDNF promote tumor-cell survival. Blood 2005, 105(11):4429-4436.

52. Kermani $P$, Hempstead B: Brain-derived neurotrophic factor: a newly described mediator of angiogenesis. Trends Cardiovasc Med 2007, 17(4):140-143.

53. Kermani $P$, Rafii D, Jin DK, Whitlock $P$, Schaffer W, Chiang A, Vincent $L$, Friedrich M, Shido K, Hackett NR, et al: Neurotrophins promote revascularization by local recruitment of TrkB+ endothelial cells and systemic mobilization of hematopoietic progenitors. J Clin Invest 2005, 115(3):653-663.

54. Kern J, Steurer M, Gastl G, Gunsilius E, Untergasser G: Vasohibin inhibits angiogenic sprouting in vitro and supports vascular maturation processes in vivo. BMC Cancer 2009, 9:284.

55. Hosaka T, Kimura H, Heishi T, Suzuki Y, Miyashita H, Ohta H, Sonoda H, Moriya T, Suzuki S, Kondo T, et al: Vasohibin-1 expression in endothelium of tumor blood vessels regulates angiogenesis. Am J Pathol 2009, 175(1):430-439.

56. Sato $Y$ : Is vasohibin-1 for more than angiogenesis inhibition? J Biochem 2011, 149(3):229-230.

57. Kimura H, Miyashita H, Suzuki Y, Kobayashi M, Watanabe K, Sonoda H, Ohta H, Fujiwara T, Shimosegawa T, Sato Y: Distinctive localization and opposed roles of vasohibin-1 and vasohibin-2 in the regulation of angiogenesis. Blood 2009, 113(19):4810-4818.

58. Lu C, Han HD, Mangala LS, Ali-Fehmi R, Newton CS, Ozbun L, Armaiz-Pena $\mathrm{GN}, \mathrm{Hu}$ W, Stone RL, Munkarah A, et al: Regulation of tumor angiogenesis by EZH2. Cancer Cell 2010, 18(2):185-197.

59. Aderem A: Systems biology: its practice and challenges. Cell 2005, 121(4):511-513.

doi:10.1186/1471-2164-14-23

Cite this article as: Affara et al:: Vasohibin-1 is identified as a masterregulator of endothelial cell apoptosis using gene network analysis. BMC Genomics 2013 14:23. 